\title{
A Computational Method of Linearization of Nonlinear Systems by Cubic Hermite Interpolation and a Nonlinear Filter
}

\author{
${ }^{\circ}$ Katsuhiro NARIKIYO $†$ and Hitoshi TAKATA $\ddagger$ \\ $\dagger$ Hiroshima National College of Maritime Technology, 4272-1, Higashino, Toyota, \\ Hiroshima 725-02, JAPAN \\ $\ddagger$ Kagoshima University, 1-21-40, Korimoto, Kagoshima 890, JAPAN
}

\begin{abstract}
A computational method of the formal linearization using a piecewise cubic Hermite interpolation is proposed. We introduce a linearizing function that consists of the state variables, their squares, and the cubes. The nonlinear terms are approximated by the method of the piecewise cubic Hermite interpolation and linearized with respect to the linearizing function. Numerical computations are easily carried out with the aid of computers. A nonlinear filter is synthesized as an application of this method and is verified through numerical examples.
\end{abstract}

\section{Introduction}

Practical system in engineering world are all inherently nonlinear process. They are most naturally described by nonlinear differential equations. The tools of linear analysis are quite well developed, while those being able to deal with nonlinear phenomena have been feeble and a few. So one wishes to have linearizing techniques of nonlinear systems so as to apply the linear theories or tools. For the last few decades, this problem has been studied using geometric methods by many researchers (e.g. $\left.{ }^{(1)}\right)$. Though many interesting results have been developed, they are, in general, not so easily applicable to practical systems. Therefore, it is necessary to study how to compute the linearization that enables easy implementation ${ }^{(2,3)}$.

In this paper we develop a numerical computational method of the formal linearization for nonlinear systems ${ }^{(2)}$. A linearizing function consists of a sequence of the terms of the cubic polynomial, i.e. state variables, their squares and cubes, thus the dimension of the linearizing function is determined automatically. The nonlinear terms are approximated by the cubic Hermite interpolation and linearized with respect to the linearizing function. As an application of this linearization, a nonlinear filter algorithm is synthesized. With the aid of computers, we easily carry out the numerical computation of transformation from a nonlinear system to a formal linear system. Results of the numerical examples of a initial value problem of differential equation and a state estimation problem show the validity of this linearization method.

\section{Formal Linearization}

\section{$2.1 \quad$ Scalar System}

Assume that a nonlinear scalar system is given by

$$
\dot{x}(t)=f(x(t)), x(0)=x_{0},
$$

where $x(t)$ is a state variable, the domain of $x(t)$ is $D=[\mu, \mu+\nu) \subset R(\mu \in R, \nu>0)$, and $f$ is a smooth nonlinear function. As a linearizing function, we introduce

$$
\phi(x)=\left[x, x^{2}, x^{3}\right]^{T} .
$$

Differentiating $\phi(x)$ with respect to $t$, we have

$$
\begin{aligned}
\dot{\phi}(x) & =\frac{d \phi}{d x} \dot{x}=\left[1,2 x, 3 x^{2}\right]^{T} f(x) \\
& \equiv\left[g_{1}(x), g_{2}(x), g_{3}(x)\right]^{T} .
\end{aligned}
$$

We approximate each element function $g_{k}(x)$ of Eq.(3) by the cubic Hermite interpolation. We divide the domain $D$ into $m$ subdomains appropriately with considering the nonlinearity of function $f(x)$. Let $D_{i}$ be the $i$ th subdomain: 


$$
D_{i}=\left[\alpha_{i}, \alpha_{i}+\delta_{i}\right),\left(\delta_{i}>0\right),
$$

where $D=\bigcup_{i=1}^{m} D_{i}$. When $x \in D_{i}$, let $h_{i k}(x)$ be a cubic Hermite interpolation of $g_{k}(x)$ :

$$
\begin{aligned}
g_{k}(x) \approx h_{i k}(x) & =\sum_{r=0}^{3} c_{i k r} x^{r} \\
& =\left[c_{i k 1}, c_{i k 2}, c_{i k 3}\right] \phi(x)+c_{i k 0} .
\end{aligned}
$$

Cubic polynomial $h_{i k}(x)$ must satisfy the conditions:

$$
\begin{array}{ll}
h_{i k}\left(\alpha_{i}\right)=g_{k}\left(\alpha_{i}\right), & h_{i k}\left(\alpha_{i}+\delta_{i}\right)=g_{k}\left(\alpha_{i}+\delta_{i}\right), \\
h_{i k}^{\prime}\left(\alpha_{i}\right)=g_{k}^{\prime}\left(\alpha_{i}\right), & h_{i k}^{\prime}\left(\alpha_{i}+\delta_{i}\right)=g_{k}^{\prime}\left(\alpha_{i}+\delta_{i}\right),
\end{array}
$$

where ${ }^{\prime} \equiv \frac{d}{d x}$. Since the number of the coefficients of Eq.(5) is 4 , the same as the number of the conditions of Eqs.(6), $h_{i k}(x)$ is uniquely determined by

$$
\begin{gathered}
{\left[\begin{array}{c}
c_{i n 0} \\
c_{i n 1} \\
c_{i n 2} \\
c_{i n 3}
\end{array}\right]=} \\
{\left[\begin{array}{rrrr}
1 & -\alpha_{i} / \delta_{i} & \alpha_{i}^{2} / \delta_{i}^{2} & -\alpha_{i}^{3} / \delta_{i}^{3} \\
0 & 1 / \delta_{i} & -2 \alpha_{i} / \delta_{i}^{2} & 3 \alpha_{i}^{2} / \delta_{i}^{3} \\
0 & 0 & 1 / \delta_{i}^{2} & -3 \alpha_{i} / \delta_{i}^{3} \\
0 & 0 & 0 & 1 / \delta_{i}^{3}
\end{array}\right]} \\
\left.\begin{array}{rrrr}
1 & 0 & 0 & 0 \\
-3 & 3 & -2 & -1 \\
2 & -2 & 1 & 1
\end{array}\right]\left[\begin{array}{c}
g_{k}\left(\alpha_{i}\right) \\
g_{k}\left(\alpha_{i}+\delta_{i}\right) \\
\delta_{i} g_{k}^{\prime}\left(\alpha_{i}\right) \\
\delta_{i} g_{k}^{\prime}\left(\alpha_{i}+\delta_{i}\right)
\end{array}\right]
\end{gathered}
$$

Applying Eq.(5) to each function $g_{k}(x), k=1,2,3$, the right-hand side of Eq.(3) is expressed in approximated linear form with respect to $\phi(x)$ :

$$
\dot{\phi}(x) \approx A_{i} \phi(x)+b_{i},
$$

where

$$
\boldsymbol{A}_{i}=\left[\begin{array}{lll}
c_{i 11} & c_{i 12} & c_{i 13} \\
c_{i 21} & c_{i 22} & c_{i 23} \\
c_{i 31} & c_{i 32} & c_{i 33}
\end{array}\right], \boldsymbol{b}_{i}=\left[\begin{array}{c}
c_{i 10} \\
c_{i 20} \\
c_{i 30}
\end{array}\right]
$$

Thus Eq.(1) is converted into the formal linear system:

$$
\dot{\boldsymbol{z}}(t)=A_{i} \boldsymbol{z}(t)+\boldsymbol{b}_{i}, \quad \boldsymbol{z}(0)=\phi(x(0)) .
$$

The inversion is obtained by Eq.(2) as follows

$$
x=[1,0,0] \phi(x) .
$$

Therefore, the approximated value of $x(t)$ through the linearization is obtained by

$$
\hat{x}(t)=[1,0,0] \boldsymbol{z}(t) .
$$

\subsection{Multi-dimensional System}

Assume that a nonlinear system is given by

$$
\dot{x}(t)=f(x(t)), x(0)=x_{0},
$$

where $\boldsymbol{x}=\left[x_{1}, \ldots, x_{n}\right]^{T}$ is an $n$ state vector, the domain of $\boldsymbol{x}$ is $D=\prod_{i=1}^{n}\left[p_{i}, p_{i}+q_{i}\right) \subset R^{n}\left(p_{i} \in R, q_{i}>0\right)$, $f=\left[f_{1}, \ldots, f_{n}\right]^{T}$ is a nonlinear vector function. As a linearizing function, we introduce

$$
\begin{aligned}
\phi(x)= & {\left[T_{(100 \cdots)}(\boldsymbol{x}), T_{(200 \cdots 0)}(\boldsymbol{x}), T_{(300 \cdots 0)}(\boldsymbol{x}),\right.} \\
& T_{(010 \cdots 0)}(\boldsymbol{x}), T_{(110 \cdots 00)}(\boldsymbol{x}), \cdots, \\
& \left.T_{\left(r_{1} r_{2} r_{3} \cdots r_{n}\right)}(\boldsymbol{x}), \cdots, T_{(333 \cdots 3)}(\boldsymbol{x})\right]^{T}
\end{aligned}
$$

where

$$
\begin{aligned}
T_{\left(r_{1} r_{2} \cdots r_{n}\right)}(\boldsymbol{x}) & =\prod_{k=1}^{n} x_{k}^{r_{k}}, \\
r_{k} & =0,1,2,3\left(r_{1}+\cdots+r_{n} \neq 0\right) .
\end{aligned}
$$

Differentiating $\phi(x)$ with respect to $t$, we have

$$
\begin{aligned}
\dot{\phi}(\boldsymbol{x}) & =\frac{\partial \phi}{\partial \boldsymbol{x}^{T}} \dot{\boldsymbol{x}}=\frac{\partial \boldsymbol{\phi}}{\partial \boldsymbol{x}^{T}} \boldsymbol{f}(\boldsymbol{x}) \\
& \equiv\left[g_{1}(\boldsymbol{x}), \ldots, g_{4^{n}-1}(\boldsymbol{x})\right]^{T} \equiv \boldsymbol{G}(\boldsymbol{x}) .
\end{aligned}
$$

We approximate each element function $g_{k}(\boldsymbol{x})$ of Eq.(15) by the cubic Hermite interpolation. We divide the domain $D$ into $m$ subdomains appropriately with considering the nonlinearity of function $\boldsymbol{f}(\boldsymbol{x})$. Let $D_{i}$ be the $i$ th subdomain:

$$
D_{i}=\prod_{k=1}^{n}\left[\alpha_{i k}, \alpha_{i k}+\delta_{i k}\right),\left(\delta_{i k}>0\right)
$$

where $D=\bigcup_{i=1}^{m} D_{i}$. When $\boldsymbol{x} \in D_{i}$, let $h_{i k}(\boldsymbol{x})$ be a cubic Hermite interpolation of $g_{k}(\boldsymbol{x})$ :

$$
g_{k}(x) \approx h_{i k}(x)=\sum_{r_{n}=0}^{3} \cdots \sum_{r_{1}=0}^{3} c_{i k\left[r_{1} \ldots r_{n}\right]} \prod_{j=1}^{n} x_{j}^{r_{j}} .
$$

Cubic polynomial $h_{i k}(\boldsymbol{x})$ must satisfy the conditions:

$$
h_{i k}^{\left(p_{1} p_{2} \cdots p_{n}\right)}(\boldsymbol{\alpha})=g_{k}^{\left(p_{1} p_{2} \cdots p_{n}\right)}(\boldsymbol{\alpha}),
$$

where

$\alpha=\left[\alpha_{i 1}+q_{1} \delta_{i 1}, \alpha_{i 2}+q_{2} \delta_{i 2}, \ldots, \alpha_{i n}+q_{n} \delta_{i n}\right]^{T}, q_{j}=$ 0 or $1, p_{j}=0$ or $1, g_{k}^{(000 \cdots 0)}(\boldsymbol{x})=g_{k}(\boldsymbol{x}), g_{k}^{(100 \cdots 0)}(\boldsymbol{x})=$ $\frac{\partial}{\partial x_{1}} g_{k}(\boldsymbol{x}), g_{k}^{(010 \cdots 0)}(\boldsymbol{x})=\frac{\partial}{\partial x_{2}} g_{k}(\boldsymbol{x}), \ldots, g_{k}^{(110 \cdots 0)}(\boldsymbol{x})=$ $\frac{\partial^{2}}{\partial x_{1} \partial x_{2}} g_{k}(x), \ldots, g_{k}^{(111 \cdots 1)}(\boldsymbol{x})=\frac{\partial^{n}}{\partial x_{1} \cdots \partial x_{n}} g_{k}(\boldsymbol{x})$.

Since the number of the coefficients of Eq.(17) is $4^{n}$, the same as the number of conditions of Eqs.(18), $h_{i k}(\boldsymbol{x})$ is uniquely determined by 


$$
c_{i}=X_{i} \Delta_{i} W^{-1} F_{i}
$$

$c_{i}$ is a $4^{n}$ dimensional vector. Let $c_{a}$ be the $a$ th element of $c_{i}$, the relation between $a$ and $r_{1}, \ldots, r_{n}$ of $c_{i k\left[r_{1} \ldots r_{n}\right]}$ is $a=\sum_{j=1}^{n}\left(4^{j-1} r_{j}\right)+1, \quad r_{j}=0,1,2$, or 3.

$F_{i}$ is a $4^{n}$ dimensional vector. Each element of $F_{i}$ is

$$
\begin{aligned}
F_{a} & =\left(\prod_{j=1}^{n} \delta_{i j}^{p_{j}}\right) g_{k}^{\left(p_{1} \ldots p_{n}\right)}\left(\left[\alpha_{i 1}+q_{1} \delta_{i 1}, \ldots, \alpha_{i n}+q_{n} \delta_{i n}\right]^{T}\right), \\
a & =\sum_{j=1}^{n}\left(2^{n+j-1} p_{j}+2^{j-1} q_{j}\right)+1 \\
p_{j} & =0 \text { or } 1, q_{j}=0 \text { or } 1 .
\end{aligned}
$$

$W$ is a $4^{n} \times 4^{n}$ dimensional matrix. Each element of $W$ is

$$
\begin{aligned}
W_{a b} & =\prod_{j=1}^{n} r_{j}^{p_{j}} q_{j}^{r_{j}-p_{j}}, \\
a & =\sum_{j=1}^{n}\left(2^{n+j-1} p_{j}+2^{j-1} q_{j}\right)+1, \\
b & =\sum_{j=1}^{n}\left(4^{j-1} r_{j}\right)+1, \\
p_{j} & =0 \text { or } 1, q_{j}=0 \text { or } 1, r_{j}=0,1,2, \text { or } 3 .
\end{aligned}
$$

$\Delta_{i}$ is a $4^{n} \times 4^{n}$ dimensional diagonal matrix. Each diagonal element of $\Delta_{i}$ is

$$
\begin{aligned}
\Delta_{a a} & =\left(\prod_{j=1}^{n} \delta_{i j}^{r_{j}}\right)^{-1}, \\
a & =\sum_{j=1}^{n}\left(4^{j-1} r_{j}\right)+1, r_{j}=0,1,2, \text { or } 3 .
\end{aligned}
$$

$X_{i}$ is a $4^{n} \times 4^{n}$ dimensional matrix:

$$
X_{i}=M\left(\alpha_{i n}\right) \otimes \cdots \otimes M\left(\alpha_{i 2}\right) \otimes M\left(\alpha_{i 1}\right),
$$

where $\otimes$ denotes tensor product. For any matrix $B$, $M(y) \otimes B$ is defined by

$$
M(y) \otimes B=\left[\begin{array}{cccc}
B & -y B & y^{2} B & -y^{3} B \\
0 & B & -2 y B & 3 y^{2} B \\
0 & 0 & B & -3 y B \\
0 & 0 & 0 & B
\end{array}\right] .
$$

Applying Eq.(17) to each function $g_{k}(\boldsymbol{x}), 1 \leq k \leq$ $4^{n}-1$, the right-hand side of Eq.(15) is expressed in approximated linear form with respect to $\phi(x)$,

$$
\dot{\phi}(x) \approx A_{i} \phi(x)+b_{i},
$$

where

$$
\begin{aligned}
& \boldsymbol{A}_{i}= \\
& {\left[\begin{array}{cccc}
c_{i 1[10 \cdots 0]} & c_{i 1[20 \cdots 0]} & \cdots & c_{i 1[33 \cdots 3]} \\
c_{i 2[10 \cdots 0]} & c_{i 2[20 \cdots 0]} & \cdots & c_{i 2[33 \cdots 3]} \\
\vdots & \vdots & \ddots & \vdots \\
c_{i 4^{n}-1[10 \cdots 0]} & c_{i 4^{n}-1[20 \cdots 0]} & \cdots & c_{i 4^{n}-1[33 \cdots 3]}
\end{array}\right],} \\
& \boldsymbol{b}_{i}=\left[c_{i 1[00 \cdots 0]}, c_{i 2[00 \cdots 0]}, \ldots, c_{i 4^{n}-1[00 \cdots 0]}\right]^{T} .
\end{aligned}
$$

Thus Eq.(12) is converted into the linear system

$$
\dot{z}(t)=A_{i} z(t)+b_{i}, z(0)=\phi\left(x_{0}\right) .
$$

The inversion is obtained by Eq.(13) as follows

$$
x=K \phi(x),
$$

where $\boldsymbol{K}$ is an $n \times\left(4^{n}-1\right)$ dimensional constant matrix and its element $k_{i j}$ is

$$
k_{i j}= \begin{cases}1 & \text { if } j=4^{i-1} \quad(1 \leq i \leq n), \\ 0 & \text { otherwise. }\end{cases}
$$

Therefore the approximated value of $x(t)$ through the linearization is obtained by

$$
\hat{\boldsymbol{x}}(t)=\boldsymbol{K} z(t) .
$$

\section{Error bound}

Let $\|\cdot\|$ be the maximum norm and $g(\boldsymbol{x})$ be one of element functions of Eq.(15). Here we introduce an operator $\Psi_{i}^{j}$ which approximates a function $g(\boldsymbol{x})$ by the cubic Hermite interpolation with respect to $x_{j}$ on subdomain $D_{i}$ such that

$$
\Psi_{i}^{j}: g(\boldsymbol{x}) \rightarrow \sum_{k=0}^{3} c_{i k}\left(\overline{\boldsymbol{x}}_{j}\right) x_{j}^{k},
$$

where

$$
\begin{aligned}
{\left[\begin{array}{c}
c_{i 0}\left(\overline{\boldsymbol{x}}_{j}\right) \\
c_{i 1}\left(\overline{\boldsymbol{x}}_{j}\right) \\
c_{i 2}\left(\overline{\boldsymbol{x}}_{j}\right) \\
c_{i 3}\left(\overline{\boldsymbol{x}}_{j}\right)
\end{array}\right]=} & {\left[\begin{array}{rrrr}
1 & -\alpha_{i j} / \delta_{i j} & \alpha_{i j}^{2} / \delta_{i j}^{2} & -\alpha_{i j}^{3} / \delta_{i j}^{3} \\
0 & 1 / \delta_{i j} & -2 \alpha_{i j} / \delta_{i j}^{2} & 3 \alpha_{i j}^{2} / \delta_{i j}^{3} \\
0 & 0 & 1 / \delta_{i j}^{2} & -3 \alpha_{i j} / \delta_{i j}^{3} \\
0 & 0 & 0 & 1 / \delta_{i j}^{3}
\end{array}\right] } \\
& {\left[\begin{array}{rrrr}
1 & 0 & 0 & 0 \\
0 & 0 & 1 & 0 \\
-3 & 3 & -2 & -1 \\
2 & -2 & 1 & 1
\end{array}\right]\left[\begin{array}{c}
g\left(\boldsymbol{y}_{1}\right) \\
g\left(\boldsymbol{y}_{2}\right) \\
\delta_{i j} g_{x_{j}}\left(\boldsymbol{y}_{1}\right) \\
\delta_{i j} g_{x_{j}}\left(\boldsymbol{y}_{2}\right)
\end{array}\right], } \\
\overline{\boldsymbol{x}}_{j}= & {\left[x_{1}, \ldots, x_{j-1}, x_{j+1}, \ldots, x_{n}\right]^{T}, } \\
\boldsymbol{y}_{1}= & {\left[x_{1}, \ldots, x_{j-1}, \alpha_{i j}, x_{j+1}, \ldots, x_{n}\right]^{T}, } \\
\boldsymbol{y}_{2}= & {\left[x_{1}, \ldots, x_{j-1}, \alpha_{i j}+\delta_{i j}, x_{j+1}, \ldots, x_{n}\right]^{T}, } \\
g_{x_{j}}= & \frac{\partial}{\partial x_{j}} g .
\end{aligned}
$$

Moreover, a product of operator, $\Psi_{i}^{[j]}$ is defined as 


$$
\Psi_{i}^{[j]} \equiv \Psi_{i}^{j} \circ \cdots \circ \Psi_{i}^{2} \circ \Psi_{i}^{1}, \quad \Psi_{i}^{[0]} g(x)=g(x) .
$$

Let $\bigcap_{i=1}^{n} C^{4}\left(x_{i}\right)$ be a set of four times continuously differentiable functions with respect to $x_{i}(i=1, \ldots, n)$. If $\boldsymbol{f}(\boldsymbol{x}) \in \bigcap_{i=1}^{n} C^{4}\left(x_{i}\right), g(\boldsymbol{x})$ also belongs to $\bigcap_{i=1}^{n} C^{4}\left(x_{i}\right)$, and an error bound of approximation by the cubic Hermite interpolation with respect to $x_{j}$ is ${ }^{(4)}$

$$
\sup _{\boldsymbol{x} \in D_{i}}\left|g(\boldsymbol{x})-\Psi_{i}^{j} g(\boldsymbol{x})\right| \leq \frac{1}{384} \delta_{i j}^{4} \sup _{\boldsymbol{x} \in D_{i}}\left|\frac{\partial^{4}}{\partial x_{j}^{4}} g(\boldsymbol{x})\right| \cdot(27)
$$

First, we have the following lemma about an error bound between $g(x)$ and $\Psi_{i}^{[n]} g(x)$.

Lemma 1: If $\boldsymbol{f}(\boldsymbol{x}) \in \bigcap_{i=1}^{n} C^{4}\left(x_{i}\right)$, the error bound of the cubic Hermite interpolation on subdomain $D_{i}$ is

$$
\sup _{x \in D_{i}}\left|g(\boldsymbol{x})-\Psi_{i}^{[n]} g(\boldsymbol{x})\right| \leq \frac{1}{384} \bar{\delta}_{i}^{4} \sum_{j=1}^{n} M_{i j}
$$

where $\bar{\delta}_{i}=\max _{1 \leq j \leq n} \delta_{i j}, M_{i j}=\sup _{\boldsymbol{x} \in D_{i}}\left|\frac{\partial^{4}}{\partial x_{j}^{4}} \Psi_{i}^{[j-1]} g(\boldsymbol{x})\right|$.

Proof: The error bound of function approximation on $D_{i}$ is

$$
\begin{aligned}
\sup _{x \in D_{i}} & \left|g(\boldsymbol{x})-\Psi_{i}^{[n]} g(\boldsymbol{x})\right| \\
& =\sup _{\boldsymbol{x} \in D_{i}}\left|\Psi_{i}^{[0]} g(\boldsymbol{x})-\Psi_{i}^{[n]} g(\boldsymbol{x})\right| \\
& \leq \sum_{j=1}^{n} \sup _{x \in D_{i}}\left|\Psi_{i}^{[j-1]} g(\boldsymbol{x})-\Psi_{i}^{[j]} g(\boldsymbol{x})\right| \\
& =\sum_{j=1}^{n} \sup _{x \in D_{i}}\left|\Psi_{i}^{[j-1]} g(\boldsymbol{x})-\Psi_{i}^{j}\left(\Psi_{i}^{[j-1]} g(\boldsymbol{x})\right)\right| .
\end{aligned}
$$

From Eq.(27),

$$
\begin{aligned}
\sup _{\boldsymbol{x} \in D_{i}} & \left|g(\boldsymbol{x})-\Psi_{i}^{[n]} g(\boldsymbol{x})\right| \\
\leq & \leq \sum_{j=1}^{n} \frac{1}{384} \delta_{i j}^{4} \sup _{\boldsymbol{x} \in D_{i}}\left|\frac{\partial^{4}}{\partial x_{j}^{4}} \Psi_{i}^{[j-1]} g(\boldsymbol{x})\right| \\
& \leq \frac{1}{384} \bar{\delta}_{i}^{4} \sum_{j=1}^{n} M_{i j} .
\end{aligned}
$$

Furthermore, an approximation of a vector function $\boldsymbol{G}(\boldsymbol{x})$ of Eq.(15) by the cubic Hermite interpolation on $D_{i}$ is expressed by

$$
\Psi_{i}^{[n]} \boldsymbol{G}(\boldsymbol{x})=\left[\Psi_{i}^{[n]} g_{1}(\boldsymbol{x}), \ldots, \Psi_{i}^{[n]} g_{4^{n}-1}(\boldsymbol{x})\right]^{T} .
$$

Let $\varepsilon_{i}(\boldsymbol{x})$ be an approximation error on $D_{i}$

$$
\varepsilon_{i}(x) \equiv G(x)-\Psi_{i}^{[n]} G(x) .
$$

We have

$$
\begin{aligned}
& \sup _{\boldsymbol{x} \in D_{i}}\left\|\varepsilon_{i}(\boldsymbol{x})\right\|=\sup _{\boldsymbol{x} \in D_{i}} \max _{1 \leq k \leq 4^{n}-1}\left|g_{k}(\boldsymbol{x})-\Psi_{i}^{[n]} g_{k}(\boldsymbol{x})\right| \\
& =\max _{1 \leq k \leq 4^{n}-1} \sup _{\boldsymbol{x} \in D_{i}}\left|g_{k}(\boldsymbol{x})-\Psi_{i}^{[n]} g_{k}(\boldsymbol{x})\right| .
\end{aligned}
$$

From lemma 1,

$$
\sup _{\boldsymbol{x} \in D_{i}}\left\|\varepsilon_{i}(\boldsymbol{x})\right\| \leq \frac{1}{384} \bar{\delta}_{i}^{4} \max _{1 \leq k \leq 4^{n}-1} \sum_{j=1}^{n} M_{i j k},
$$

where $M_{i j k}=\sup _{x \in D_{i}}\left|\frac{\partial^{4}}{\partial x_{j}^{4}} \Psi^{[j-1]} g_{k}(x)\right|$.

Therefore, we have the following lemma about an error bound of the vector function approximation.

Lemma 2: If $\boldsymbol{f}(\boldsymbol{x}) \in \bigcap_{i=1}^{n} C^{4}\left(x_{i}\right)$,

$$
\varepsilon \equiv \max _{1 \leq i \leq m} \sup _{\boldsymbol{x} \in D_{i}}\left\|\varepsilon_{i}(\boldsymbol{x})\right\| \leq \frac{M}{384} \bar{\delta}^{4},
$$

where $\bar{\delta}=\max _{1 \leq i \leq m} \bar{\delta}_{i}, M=\max _{1 \leq i \leq m} \max _{1 \leq k \leq 4^{n}-1} \sum_{j=1}^{n} M_{i j k}$.

Finally, we have the following theorem about an error bound between $\boldsymbol{x}(t)$ and $\hat{x}(t)$.

Theorem: An error bound of the formal linearization is

$$
\begin{aligned}
& \|x(t)-\hat{x}(t)\| \\
& \leq e^{a t}\|\phi(x(0))-\boldsymbol{z}(0)\|+\varepsilon\left(e^{a t}-1\right) / a,
\end{aligned}
$$

where

$$
\begin{aligned}
a & =\max _{1 \leq i \leq m}\left\|A_{i}\right\|, \\
\varepsilon & =\max _{1 \leq i \leq m} \sup _{\boldsymbol{x} \in D_{i}}\left\|\varepsilon_{i}(\boldsymbol{x})\right\| \\
& \leq \frac{M}{384} \bar{\delta}^{4} \quad\left(\text { if } \boldsymbol{f}(\boldsymbol{x}) \in \bigcap_{i=1}^{n} C^{4}\left(x_{i}\right)\right) .
\end{aligned}
$$

Proof: If $x(t) \in D_{i}$ for any $i$, a differential equation of difference between $\boldsymbol{\phi}(\boldsymbol{x})$ of Eq.(13) and $\boldsymbol{z}$ of Eq.(21) is given by

$$
\begin{aligned}
& \frac{d}{d t}(\phi(x)-z)=\dot{\phi}(x)-\dot{z}=G(x)-A_{i} z-b_{i} \\
& =A_{i}(\phi(x)-z)+\left(G(x)-A_{i} \phi(x)-b_{i}\right) .
\end{aligned}
$$

The solution of this equation is

$$
\begin{aligned}
\phi(x(t))-\boldsymbol{z}(t) & =e^{\boldsymbol{A}_{i} t}[\phi(x(0))-\boldsymbol{z}(0)] \\
& +\int_{0}^{t} e^{\boldsymbol{A}_{i}(t-\tau)} \varepsilon_{i}(\boldsymbol{x}(\tau)) d \tau .
\end{aligned}
$$


The norm of Eq.(39) is

$$
\begin{aligned}
& \|\phi(\boldsymbol{x}(t))-\boldsymbol{z}(t)\| \\
& \leq e^{\left\|\boldsymbol{A}_{i}\right\| t}\|\phi(\boldsymbol{x}(0))-\boldsymbol{z}(0)\| \\
& \quad+\sup _{\boldsymbol{x} \in D_{i}}\left\|\varepsilon_{i}(\boldsymbol{x})\right\| \int_{0}^{t} e^{\left\|\boldsymbol{A}_{i}\right\|(t-\tau)} d \tau \\
& \leq e^{a t}\|\phi(\boldsymbol{x}(0))-\boldsymbol{z}(0)\|+\varepsilon \int_{0}^{t} e^{a(t-\tau)} d \tau \\
& =e^{a t}\|\phi(\boldsymbol{x}(0))-\boldsymbol{z}(0)\|+\varepsilon\left(e^{a t}-1\right) / a .
\end{aligned}
$$

Eq.(40) is valid on all subdomains. From Eqs.(22) and (24),

$$
\begin{aligned}
& \|\boldsymbol{x}(t)-\hat{x}(t)\|=\|\boldsymbol{K} \phi(\boldsymbol{x}(t))-\boldsymbol{K} z(t)\| \\
& \leq\|\boldsymbol{K}\|\|\phi(\boldsymbol{x}(t))-\boldsymbol{z}(t)\| \\
& =\|\phi(x(t))-\boldsymbol{z}(t)\| .
\end{aligned}
$$

Eqs. (40) and (41) yield Eq.(36).

Theorem indicates that an initial error causes the first term, and the approximation error by the Hermite interpolation dose the second term which can be reduced when $\bar{\delta}$ is reduced. Therefore, if $z(0)=\phi(0)$ and $\bar{\delta} \rightarrow 0$, then $\hat{x}(t) \rightarrow x(t)$ at any time $t$

\section{A Nonlinear Filter}

We here synthesize a nonlinear filter as an application of the linearization. Assume that a nonlinear dynamic and a nonlinear measurement equations are given as

$$
\begin{aligned}
\dot{x}(t) & =f(x(t)), x(0)=x_{0}, \\
\zeta(t) & =h(x(t))+v(t),
\end{aligned}
$$

where $\zeta=\left[\zeta_{1}, \ldots, \zeta_{r}\right]^{T}: r$ measurement vector, $h=$ $\left[h_{1}, \ldots, h_{r}\right]^{T}: r$ nonlinear measurement vector function, $v(\mathrm{t})$ : white Gaussian noise of $E(v(t))=0$ and $E\left(v(t) v^{T}(\tau)\right)=V \delta(t-\tau), \delta$ :Dirac delta function.

Eq.(42) is transformed into the linear system of Eq.(21) by the formal linearization. For Eq.(43), approximate it by the cubic Hermite interpolation

$$
h(x) \approx C_{i} \phi(x)+d_{i}, x \in D_{i} .
$$

Thus we have linearized dynamic and measurement equations:

$$
\begin{aligned}
\dot{\boldsymbol{z}} & =A_{i} z(t)+b_{i}, \\
\zeta(t) & =C_{i} z(t)+d_{i}+v(t) .
\end{aligned}
$$

We apply the linear estimation theory, Kalman filter ${ }^{(5)}$, to Eqs.(45) and (46). and have a nonlinear filter algorithm:

$$
\begin{aligned}
\dot{\hat{z}}= & \left(A_{i}-L C_{i}\right) \hat{z}+b_{i}+L\left(\zeta-d_{i}\right), \\
L= & P C_{i}^{T} V^{-1}, \\
\dot{P}= & A_{i} P+P A_{i}^{T}-P C_{i}^{T} V^{-1} C_{i} P, \\
\hat{x}= & K \hat{z} \in D_{i}, \\
& \hat{x}\left(t_{0}\right), P\left(t_{0}\right) \text { : given. }
\end{aligned}
$$

Matrices $\boldsymbol{A}_{i}, \boldsymbol{b}_{i}, \boldsymbol{C}_{i}$ and $\boldsymbol{d}_{i}$ must be changed into $\boldsymbol{A}_{j}$, $\boldsymbol{b}_{j}, \boldsymbol{C}_{j}$ and $\boldsymbol{d}_{j}$ respectively, when $\hat{\boldsymbol{x}}(t)$ goes onto different subdomain $D_{j}$ from $D_{i}$ during state estimation.

\section{Numerical Examples}

We have numerical experiments of the formal linearization and the nonlinear filter. Differential equations are solved by Runge-Kutta method.

Example 1: Consider the following two dimensional nonlinear system:

$$
\left\{\begin{array}{l}
\dot{x}_{1}=-1000\left(x_{1}-x_{2}^{4}\right)+4 x_{2}^{3}, \\
\dot{x}_{2}=1,
\end{array}\right.
$$

where the initial value is $x(0)=[0,0]^{T}$. The analytic solution of this system is $x_{1}(t)=t^{4}, x_{2}(t)=t$. To apply our linearization method, set the domain as $x \in[-1,1) \times[-1,1)$, and let $N$ be a parameter such as $\delta_{i k}=2^{-N}\left(m=4^{2 N}, 1 \leq i \leq m, k=1,2\right)$ of Eq.(16). For the purpose of comparison, we apply Runge-Kutta method to the original nonlinear system of Eq.(51) with time step $\Delta t=0.001$. Fig. 1 shows the trajectories of $x_{1}(t)$ and $\hat{x}_{1}(t)$, and Fig. 2 shows the error defined as

$$
J(t)=\int_{0}^{t}\|\boldsymbol{x}(\tau)-\hat{x}(\tau)\| d \tau .
$$

Those results of Figs. 1 and 2 show that the accuracy of the formal linearization is improved for smaller size of subdomains.

Example 2: Consider the following nonlinear system:

$$
\begin{aligned}
& \dot{x}(t)=\cos (x(t)), x(0)=0.0, \\
& \zeta(t)=\sin \frac{x(t)}{2}+v(t), \quad V=0.2 .
\end{aligned}
$$


To apply our nonlinear filter algorithm to the system, set the domain of $x$ as $[0,2)$, and $\delta_{i k}=0.5(m=$ 4, $1 \leq i \leq m, k=1)$. Give the initial values $\hat{x}(0)=1.5, P(0)=5 I$. For the purpose of comparison, we apply the extended Kalman filter ${ }^{(5)}$ to the above system with initial values $\hat{x}(0)=1.5, P(0)=5$. Fig. 3 shows the results of state estimation. These results indicate that the estimation by the proposed filter is better than the result by the extended Kalman filter.

\section{Conclusions}

A numerical computational method of the formal linearization by the cubic Hermite interpolation is considered. This linearization method is applicable to practical nonlinear systems and easily computable with the aid of computers. Based on this method, a new nonlinear filter algorithm is derived. Finally the linearization and the nonlinear filter algorithm are validated through numerical experiments.

\section{References}

[1] A. Isidori: Nonlinear Control Systems, SpringerVerlag (1985)

[2] H. Takata: Transformation of a Nonlinear Systems into an Augmented Linear System, IEEE Trans. Autom. Control, 24, No.5, 736/741 (1979)

[3] K. Komatsu, H. Takata, \& T. Tsuji: A Formal Linearization of Nonlinear Systems by the Chebyshev Interpolation and a Nonlinear Filter as an Application, IEICE Trans. Fundamentals, E77A, No.11, 1753/1757 (1994)

[4] Martin H. Schultz: Spline Analysis, Prentice-Hall, Inc. (1973)

[5] A. P. Sage \& C. C. White III: Optimum System Control, 2nd edition, Prentice-Hall, Inc. (1977)

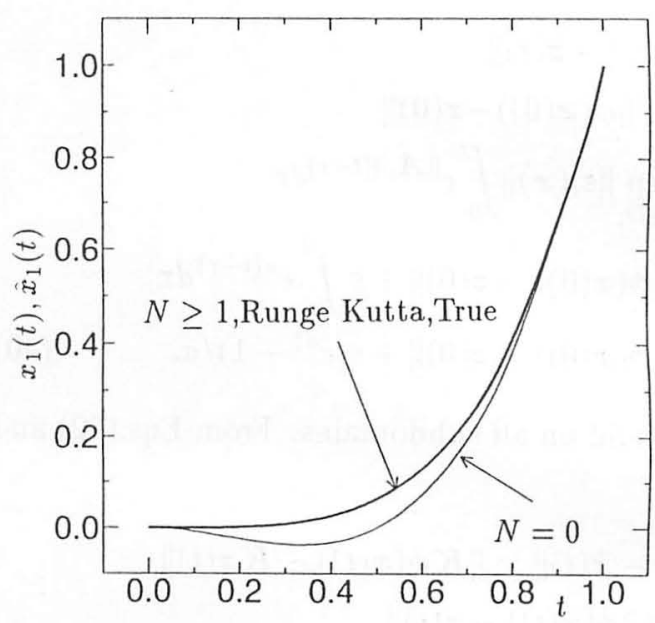

Fig. 1: Trajectories of $x_{1}(t)$ and $\hat{x}_{1}(t)$.

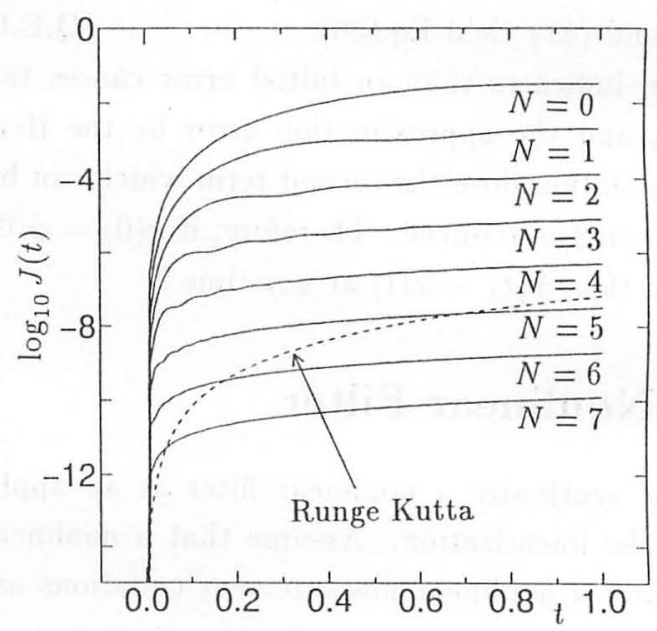

Fig. 2: Approximation errors $J(t)$.

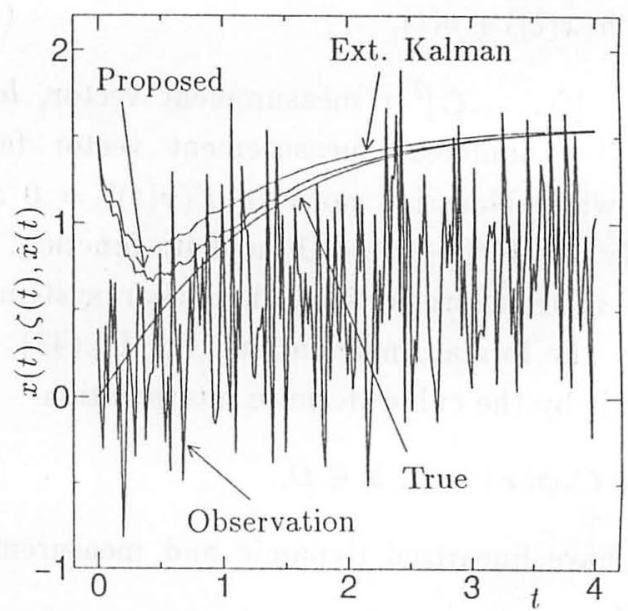

Fig. 3: Estimation results. 